\title{
Long-range quadrupole electron-phonon interaction from first principles
}

\author{
Jinsoo Park $\odot,{ }^{1}$ Jin-Jian Zhou $\odot,{ }^{1}$ Vatsal A. Jhalani $\odot,{ }^{1}$ Cyrus E. Dreyer, ${ }^{2,3}$ and Marco Bernardi $\oplus^{1, *}$ \\ ${ }^{1}$ Department of Applied Physics and Materials Science, California Institute of Technology, Pasadena, California 91125, USA \\ ${ }^{2}$ Department of Physics and Astronomy, Stony Brook University, Stony Brook, New York 11794-3800, USA \\ ${ }^{3}$ Center for Computational Quantum Physics, Flatiron Institute, 162 Fifth Avenue, New York 10010, USA
}

(Received 30 March 2020; accepted 29 May 2020; published 21 September 2020)

\begin{abstract}
Lattice vibrations in materials induce perturbations on the electron dynamics in the form of long-range (dipole and quadrupole) and short-range (octopole and higher) potentials. The dipole Fröhlich term can be included in current first-principles electron-phonon (e-ph) calculations and is present only in polar materials. The quadrupole e-ph interaction is present in both polar and nonpolar materials, but currently it cannot be computed from first principles. Here we show an approach to compute the quadrupole e-ph interaction and include it in $a b$ initio calculations of e-ph matrix elements. The accuracy of the approach is demonstrated by comparing with direct density functional perturbation theory calculations. We apply our method to silicon as a case of a nonpolar semiconductor and tetragonal $\mathrm{PbTiO}_{3}$ as a case of a polar piezoelectric material. In both materials we find that the quadrupole term strongly impacts the e-ph matrix elements. Analysis of e-ph interactions for different phonon modes reveals that the quadrupole term mainly affects optical modes in silicon and acoustic modes in $\mathrm{PbTiO}_{3}$, although the quadrupole term is needed for all modes to achieve quantitative accuracy. The effect of the quadrupole e-ph interaction on electron scattering processes and transport is shown to be important. Our approach enables accurate studies of e-ph interactions in broad classes of nonpolar, polar, and piezoelectric materials.
\end{abstract}

DOI: 10.1103/PhysRevB.102.125203

\section{INTRODUCTION}

Electron-phonon (e-ph) interactions are key to understanding electrical transport, nonequilibrium dynamics, and superconductivity [1]. First-principles calculations can provide microscopic insight into e-ph scattering processes and are rapidly emerging as a quantitative tool for investigating charge transport and ultrafast carrier dynamics in materials [2-13]. The typical workflow combines density functional theory (DFT) [14] calculations of the ground state and band structure with density functional perturbation theory (DFPT) [15] for phonon dispersions and e-ph perturbation potentials. As DFPT can compute the electronic response to periodic lattice perturbations (phonons) with arbitrary wave-vector $\boldsymbol{q}$, the DFPT framework can capture both short- and long-range e-ph interactions.

However, a key challenge is that DFPT is too computationally demanding to be carried out on the fine Brillouin zone grids needed to compute electron scattering rates and transport properties. The established approach in first-principles e-ph studies [9] is to carry out DFPT calculations on coarse Brillouin zone grids with order of $10 \times 10 \times 10 q$ points, followed by interpolation of the e-ph matrix elements with a localized basis set such as Wannier functions or atomic orbitals [16]. As the perturbation potential can be nonanalytic near $\boldsymbol{q}=0$ [16] or even exhibit a divergence for certain phonon modes, interpolation is particularly challenging and less reliable in the region between $\boldsymbol{q}=0$ and its nearest-neighbor $\boldsymbol{q}$ points

*Corresponding author: bmarco@caltech.edu in the coarse DFPT grid. This small- $\boldsymbol{q}$ region is critical as it is dominated by long-range e-ph interactions, whose treatment can affect the quality of the interpolation even at larger values of $q$ in the Brillouin zone.

A multipole expansion of the e-ph perturbation potential shows that in the long-wavelength limit (phonon wave-vector $\boldsymbol{q} \rightarrow 0$ ) the long-range dipole Fröhlich term diverges as $1 / q$, the quadrupole term approaches a constant value and the short-range octopole and higher terms vanish $[17,18]$. These trends in momentum space are due to the spatial decay of the e-ph interactions, with $1 / r^{2}$ trend for the dipole, $1 / r^{3}$ for the quadrupole, and $1 / r^{4}$ or faster for the short-range part. The open question is how one can carry out the eph matrix element interpolation in the region near $\boldsymbol{q}=0$ using analytical expressions for the long-range dipole and quadrupole terms. These expressions have been obtained by Vogl [18], but need to be rewritten in the $a b$ initio formalism and computed with first-principles quantities such as the atomic dynamical dipoles [15] and quadrupoles [19-21] induced by lattice vibrations, which can be computed with DFPT. For each atom $\kappa$, one can obtain Born charge $\left(\mathbf{Z}_{\kappa}\right)$ and dynamical quadrupole $\left(\mathbf{Q}_{k}\right)$ tensors, which, once contracted with the phonon eigenvector, give the atomic contributions to the dipole and quadrupole e-ph interactions.

The dipole Fröhlich term has been derived following this strategy $[22,23]$ and employed in electron scattering rate and transport calculations $[4,7]$. The quadrupole term has not yet been derived or implemented in first-principles calculations and its important effect on the e-ph matrix elements has been overlooked. To understand the role of long-range dipole and quadrupole e-ph interactions, it is useful to consider 
separately the interactions for different phonon modes in the long-wavelength limit, discerning the effect of longitudinal and transverse, and acoustic and optical modes. Analytical models of e-ph interactions rely on such an intuition for the role of different phonon modes in various materials [24].

In ionic and polar covalent crystals (here and below, denoted as polar materials), the dipole Fröhlich term is dominant as $\boldsymbol{q} \rightarrow 0$ due to its $1 / q$ trend. This e-ph interaction is due to longitudinal optical (LO) phonons and it dominates small- $\boldsymbol{q}$ scattering. For other phonon modes in polar materials, the dipole term vanishes, and the dominant long-range e-ph interaction is the quadrupole term, which is particularly important for acoustic phonons in piezoelectric (polar noncentrosymmetric) materials. In nonpolar semiconductors such as silicon and germanium, the dipole Fröhlich interaction vanishes and the quadrupole term is the dominant long-range e-ph interaction for all modes. The quadrupole e-ph interaction is thus expected to play an important role in many classes of materials, making a compelling case for its inclusion in the first-principles framework.

Here we show $a b$ initio calculations of the long-range quadrupole e-ph interaction and an approach to include it in the e-ph matrix elements. The accuracy of our method is confirmed by comparing the e-ph matrix elements with direct DFPT calculations. We find that the quadrupole contribution is significant for most phonon modes in both nonpolar and polar materials. In silicon, a nonpolar semiconductor, the quadrupole term has a large effect on the e-ph coupling for optical modes, but is negligible for acoustic modes in the long-wavelength limit. In tetragonal $\mathrm{PbTiO}_{3}$, a polar piezoelectric material, the quadrupole corrections are substantial for all phonon modes and are particularly important for acoustic modes, which contribute to the piezoelectric e-ph interaction. Including only the long-range Fröhlich interaction and neglecting the quadrupole term leads to large errors in $\mathrm{PbTiO}_{3}$, while adding the quadrupole term leads to e-ph matrix elements that accurately reproduce the DFPT benchmark results for all phonon modes in the entire Brillouin zone. We investigate the impact of the quadrupole e-ph interaction on the electron scattering rates and mobility in silicon and $\mathrm{PbTiO}_{3}$, finding mobility corrections of order $10 \%$ in silicon and $20 \%$ in $\mathrm{PbTiO}_{3}$ at $100 \mathrm{~K}$ (and smaller corrections at $300 \mathrm{~K}$ ) when the quadrupole term is included. The correction on the scattering rate at low electron energy in $\mathrm{PbTiO}_{3}$ is substantial. Taken together, our results highlight the need to include the quadrupole term in all materials to correctly capture the long-range e-ph interactions. In turn, this development enables more precise calculations of electron dynamics and scattering processes from first principles.

\section{THEORY}

The electron distribution changes in response to a displacement of an atom from its equilibrium position. The cell-integrated charge response to a displacement of atom $\kappa$ due to a phonon with wave-vector $\boldsymbol{q} \rightarrow 0$ can be written as a multipole expansion [21]:

$$
C_{\kappa, \alpha}^{q}=-i Z_{\kappa, \alpha \beta} q_{\beta}-\frac{1}{2} Q_{\kappa, \alpha \beta \gamma} q_{\beta} q_{\gamma}+\cdots,
$$
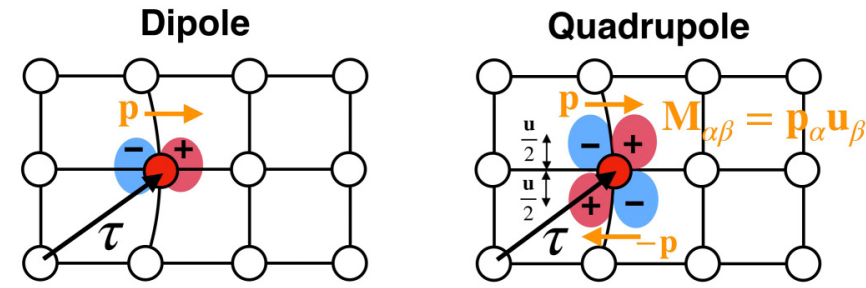

FIG. 1. Schematic of the dipole and quadrupole charge configurations giving rise to long-range e-ph interactions.

where summation over the Cartesian indices $\beta$ and $\gamma$ is implied. This polarization response defines the Born effective charge $\mathbf{Z}_{\kappa}$, a rank-2 tensor associated with the dipole term, and the dynamical quadrupole $\mathbf{Q}_{\kappa}$, the rank-3 tensor in the quadrupole term; both tensors can be computed in the DFPT framework [15,19]. Each of the dipole and quadrupole responses generates macroscopic electric fields and corresponding long-range e-ph interactions in semiconductors and insulators [18,25], while in metals they are effectively screened out.

In a field-theoretic treatment of the e-ph interactions, one computes the dipole and quadrupole perturbation potentials $\Delta V_{v \boldsymbol{q}}$ due to a phonon with mode index $v$ and wave-vector $\boldsymbol{q}$, and the corresponding e-ph matrix elements [2]

$$
g_{m n v}(\boldsymbol{k}, \boldsymbol{q})=\left(\frac{\hbar}{2 \omega_{v \boldsymbol{q}}}\right)^{1 / 2}\left\langle m \boldsymbol{k}+\boldsymbol{q}\left|\Delta V_{\nu \boldsymbol{q}}\right| n \boldsymbol{k}\right\rangle,
$$

which quantify the probability amplitude of an electron in a Bloch state $|n \boldsymbol{k}\rangle$ with band index $n$ and crystal momentum $\boldsymbol{k}$ to scatter into a final state $|m \boldsymbol{k}+\boldsymbol{q}\rangle$ by emitting or absorbing a phonon with energy $\hbar \omega_{\nu q}$.

\section{A. Dipole and quadrupole e-ph interactions}

To derive the dipole and quadrupole perturbation potentials, we consider a Born-von Karman (BvK) crystal [26] with $N$ unit cells and volume $N \Omega$. The potential due to a dipole configuration with dipole moment $\mathbf{p}$ centered at position $\boldsymbol{\tau}$ in the crystal (see Fig. 1) can be written as [22,23]

$$
\Delta V^{\mathrm{dip}}(\mathbf{r} ; \boldsymbol{\tau})=i \frac{e}{N \Omega \varepsilon_{0}} \sum_{\boldsymbol{q}} \sum_{\mathbf{G} \neq-\boldsymbol{q}} \frac{\mathbf{p} \cdot(\boldsymbol{q}+\mathbf{G}) e^{i(\boldsymbol{q}+\mathbf{G}) \cdot(\mathbf{r}-\boldsymbol{\tau})}}{(\boldsymbol{q}+\mathbf{G}) \cdot \boldsymbol{\epsilon} \cdot(\boldsymbol{q}+\mathbf{G})}
$$

where $\epsilon$ is the dielectric tensor of the material, the phonon wave-vector $\boldsymbol{q}$ belongs to a regular Brillouin zone grid with $N$ points, and $\mathbf{G}$ are reciprocal lattice vectors. This result is derived by adding together the potentials generated in the crystal by two point charges of opposite sign with distance $\mathbf{u} \rightarrow 0$, resulting in a dipole $\mathbf{p}[4,23]$.

The potential in Eq. (3) is readily extended to the case of an atomic dynamical dipole $\mathbf{p}_{\kappa, \mathbf{R}}$ from atom $\kappa$ in the unit cell at Bravais lattice vector $\mathbf{R}$, due to the displacement induced by a phonon with mode index $v$ and wave-vector $\boldsymbol{q}$. The resulting atomic dynamical dipole is $\mathbf{p}_{\kappa, \mathbf{R}}=\left(e \mathbf{Z}_{\kappa}\right) \tilde{\mathbf{e}}_{v \boldsymbol{q}}^{(\kappa)} e^{i q \cdot \mathbf{R}}$, where the phonon eigenvector projected on atom $\kappa$ is defined as $\tilde{\mathbf{e}}_{v q}^{(\kappa)}=$ $\mathbf{e}_{v q}^{(\kappa)} / \sqrt{M_{\kappa}}$, with $\mathbf{e}_{v q}$ the eigenvector of the dynamical matrix at $q$ and $M_{\kappa}$ the mass of atom $\kappa$. Summing over the contributions 
from all atoms $\kappa$ at lattice vectors $\mathbf{R}$ with positions $\boldsymbol{\tau}_{\kappa \mathbf{R}}=$ $\boldsymbol{\tau}_{\kappa}+\mathbf{R}$ in the BvK supercell, the total e-ph dipole interaction due to the phonon mode is $\Delta V_{\nu \boldsymbol{q}}^{\mathrm{dip}}(\mathbf{r})=\sum_{\kappa \mathbf{R}} \Delta V^{\mathrm{dip}}\left(\mathbf{r} ; \boldsymbol{\tau}_{\kappa \mathbf{R}}\right)$. Using the identity $\frac{1}{N} \sum_{\mathbf{R}} e^{i \boldsymbol{q} \cdot \mathbf{R}}=\delta_{\boldsymbol{q}, 0}$, we obtain

$$
\begin{aligned}
\Delta V_{\nu \boldsymbol{q}}^{\mathrm{dip}}(\mathbf{r})= & i \frac{e^{2}}{\Omega \varepsilon_{0}} \sum_{\kappa} M_{\kappa}^{-1 / 2} \sum_{\mathbf{G} \neq-\boldsymbol{q}} \\
& \times \frac{\left(\mathbf{Z}_{\kappa} \mathbf{e}_{\nu \boldsymbol{q}}^{(\kappa)}\right) \cdot(\boldsymbol{q}+\mathbf{G}) e^{i(\boldsymbol{q}+\mathbf{G}) \cdot(\mathbf{r}-\boldsymbol{\tau})}}{(\boldsymbol{q}+\mathbf{G}) \cdot \boldsymbol{\epsilon} \cdot(\boldsymbol{q}+\mathbf{G})} .
\end{aligned}
$$

The $a b$ initio Fröhlich e-ph coupling is obtained by evaluating the matrix elements with this potential:

$$
\begin{aligned}
g_{m n v}^{\operatorname{dip}}(\boldsymbol{k}, \boldsymbol{q})= & i \frac{e^{2}}{\Omega \varepsilon_{0}} \sum_{\kappa}\left(\frac{\hbar}{2 \omega_{v \boldsymbol{q}} M_{\kappa}}\right)^{1 / 2} \sum_{\mathbf{G} \neq-\boldsymbol{q}} \\
& \times \frac{\left(\mathbf{Z}_{\kappa} \mathbf{e}_{\nu \boldsymbol{q}}^{(\kappa)}\right) \cdot(\boldsymbol{q}+\mathbf{G})}{(\boldsymbol{q}+\mathbf{G}) \cdot \boldsymbol{\epsilon} \cdot(\boldsymbol{q}+\mathbf{G})} \\
& \times\left\langle m \boldsymbol{k}+\boldsymbol{q}\left|e^{i(\boldsymbol{q}+\mathbf{G}) \cdot(\mathbf{r}-\boldsymbol{\tau})}\right| n \boldsymbol{k}\right\rangle .
\end{aligned}
$$

The potential due to the dynamical quadrupole response can be derived with a similar strategy. We first consider the potential generated by a quadrupole charge configuration consisting of two equal and oppositely oriented dipoles $\mathbf{p}$ and $-\mathbf{p}$, centered at positions $\boldsymbol{\tau} \pm \frac{\mathbf{u}}{2}$, respectively (see Fig. 1). The configuration, with quadrupole moment [27] $M_{\alpha \beta}=p_{\alpha} u_{\beta}$, gives a potential:

$$
\begin{aligned}
\Delta V^{\text {quad }}(\mathbf{r} ; \boldsymbol{\tau})= & \lim _{\mathbf{u} \rightarrow 0}\left[\Delta V^{\operatorname{dip}}\left(\mathbf{r} ; \boldsymbol{\tau}+\frac{\mathbf{u}}{2}\right)-\Delta V^{\operatorname{dip}}\left(\mathbf{r} ; \boldsymbol{\tau}-\frac{\mathbf{u}}{2}\right)\right] \\
= & \frac{e}{N \Omega \varepsilon_{0}} \sum_{\boldsymbol{q}} \sum_{\mathbf{G} \neq-\boldsymbol{q}} \frac{(\boldsymbol{q}+\mathbf{G}) \cdot \mathbf{M} \cdot(\boldsymbol{q}+\mathbf{G})}{(\boldsymbol{q}+\mathbf{G}) \cdot \boldsymbol{\epsilon} \cdot(\boldsymbol{q}+\mathbf{G})} \\
& \times e^{i(\boldsymbol{q}+\mathbf{G}) \cdot(\mathbf{r}-\boldsymbol{\tau})},
\end{aligned}
$$

where to obtain the second line we used $\Delta V^{\operatorname{dip}}(\mathbf{r} ; \boldsymbol{\tau})$ in Eq. (3) and expanded the first line to first order in $\mathbf{u}$.

Similar to the dipole case, the potential from atomic quadrupoles $\left(\mathbf{M}_{\kappa, \mathbf{R}}\right)_{\alpha \beta}=\frac{1}{2}\left(e \mathbf{Q}_{\kappa}\right)_{\alpha \beta \gamma} \mathbf{e}_{\nu \boldsymbol{q}, \gamma}^{(\kappa)} e^{i \boldsymbol{q} \cdot \mathbf{R}}$ due to the displacement induced by a phonon is obtained as $\Delta V_{v \boldsymbol{q}}^{\text {quad }}(\mathbf{r})=$ $\sum_{\kappa \mathbf{R}} \Delta V^{\text {quad }}\left(\mathbf{r} ; \boldsymbol{\tau}_{\kappa \mathbf{R}}\right)$. Following steps analogous to the dipole case, we find

$$
\begin{aligned}
\Delta V_{v \boldsymbol{q}}^{\mathrm{quad}}(\mathbf{r})= & \frac{e^{2}}{\Omega \varepsilon_{0}} \sum_{\kappa} M_{\kappa}^{-1 / 2} \\
& \times \sum_{\mathbf{G} \neq-\boldsymbol{q}} \frac{1}{2} \frac{(\boldsymbol{q}+\mathbf{G}) \cdot\left(\mathbf{Q}_{\kappa} \mathbf{e}_{\nu \boldsymbol{q}}^{(\kappa)}\right) \cdot(\boldsymbol{q}+\mathbf{G})}{(\boldsymbol{q}+\mathbf{G}) \cdot \boldsymbol{\epsilon} \cdot(\boldsymbol{q}+\mathbf{G})} \\
& \times e^{i(\boldsymbol{q}+\mathbf{G}) \cdot\left(\mathbf{r}-\boldsymbol{\tau}_{\kappa}\right)} .
\end{aligned}
$$

The corresponding e-ph matrix elements due to the quadrupole perturbation potential are

$$
\begin{aligned}
g_{m n v}^{\mathrm{quad}}(\boldsymbol{k}, \boldsymbol{q})= & \frac{e^{2}}{\Omega \varepsilon_{0}} \sum_{\kappa}\left(\frac{\hbar}{2 \omega_{\nu \boldsymbol{q}} M_{\kappa}}\right)^{1 / 2} \sum_{\boldsymbol{G} \neq-\boldsymbol{q}} \frac{1}{2} \\
& \times \frac{(\boldsymbol{q}+\boldsymbol{G})_{\alpha}\left(Q_{\kappa, \alpha \beta \gamma} \mathrm{e}_{\nu \boldsymbol{q}, \gamma}^{(\kappa)}\right)(\boldsymbol{q}+\boldsymbol{G})_{\beta}}{(\boldsymbol{q}+\boldsymbol{G})_{\alpha} \epsilon_{\alpha \beta}(\boldsymbol{q}+\boldsymbol{G})_{\beta}} \\
& \times\left\langle m \boldsymbol{k}+\boldsymbol{q}\left|e^{i(\boldsymbol{q}+\mathbf{G}) \cdot\left(\mathbf{r}-\boldsymbol{\tau}_{\kappa}\right)}\right| n \boldsymbol{k}\right\rangle .
\end{aligned}
$$

Note that in the $\boldsymbol{q} \rightarrow 0$ limit the Fröhlich e-ph matrix elements are of order $1 / q$ and the quadrupole matrix elements of order $q^{0}$, thus approaching a constant value; both quantities are nonanalytic as $\boldsymbol{q} \rightarrow 0$. Octopole and higher electronic responses in Eq. (1) lead to potentials that vanish as $\boldsymbol{q} \rightarrow 0$ and can be grouped together into a short-range e-ph interaction, commonly referred to as the "deformation potential" in analytic e-ph theories [18].

\section{B. Interpolation scheme for e-ph interactions}

The total e-ph matrix elements $g$ (here we omit the band and mode indices) can be formed by adding together the short-range part $g^{\mathrm{S}}$ and the dipole and quadrupole interactions, which can be combined into a long-range part $g^{\mathrm{L}}$. Therefore,

$$
\begin{aligned}
g & =g^{\mathrm{S}}+g^{\mathrm{L}} \\
& =g^{\mathrm{S}}+g^{\text {dip }}+g^{\text {quad }} .
\end{aligned}
$$

We start from a set of e-ph matrix elements $g(\boldsymbol{k}, \boldsymbol{q})$ computed with DFPT on a regular coarse grid of $\boldsymbol{k}$ and $\boldsymbol{q}$ points [15]. The short-range part is obtained by subtracting the long-range terms on the coarse grid, $g^{\mathrm{S}}(\boldsymbol{k}, \boldsymbol{q})=g(\boldsymbol{k}, \boldsymbol{q})-$ $g^{\mathrm{dip}}(\boldsymbol{k}, \boldsymbol{q})-g^{\text {quad }}(\boldsymbol{k}, \boldsymbol{q})$. The short-range e-ph matrix elements decay rapidly in real space, and thus are ideal for interpolation using a localized basis set such as Wannier functions [28] or atomic orbitals [16]. After interpolating the short-ranged part [9] on fine $\boldsymbol{k}$ - and $\boldsymbol{q}$-point grids, we add back the longrange dipole and quadrupole matrix elements, computed using Eqs. (5) and (8) directly at the fine-grid $\boldsymbol{k}$ and $\boldsymbol{q}$ points.

As DFPT accurately captures the long-range dipole and quadrupole e-ph interactions [15], the matrix elements obtained from DFPT can be used as a benchmark for the interpolated results. For this comparison, following Ref. [22] we compute the gauge-invariant e-ph coupling strength $D_{\text {tot }}^{v}(\boldsymbol{q})$, which is proportional to the absolute value of the e-ph matrix elements:

$$
D_{\mathrm{tot}}^{v}(\boldsymbol{q})=\sqrt{\frac{2 \omega_{v \boldsymbol{q}} M_{\mathrm{uc}}}{\hbar^{2}} \sum_{m n} \frac{\left|g_{m n v}(\boldsymbol{k}=\Gamma, \boldsymbol{q})\right|^{2}}{N_{b}}},
$$

where $M_{\mathrm{uc}}$ is the mass of the unit cell and the band indices $n$ and $m$ run over the $N_{b}$ bands selected for the analysis.

\section{Computational details}

We investigate the effect of the quadrupole e-ph interaction in silicon, a nonpolar semiconductor, and tetragonal $\mathrm{PbTiO}_{3}$, a polar piezoelectric material. Calculations on $\mathrm{GaN}$ are shown in the companion work [29]. The ground state and band structure are obtained using DFT in the local density approximation with a plane-wave basis using the QUANTUM ESPRESSO code [30]. Kinetic energy cutoffs of $40 \mathrm{Ry}$ for silicon and 76 Ry for $\mathrm{PbTiO}_{3}$ are employed, together with scalarrelativistic norm-conserving pseudopotentials from pseudo Dojo [31]. We have verified that spin-orbit coupling has a negligible effect. The calculations employ relaxed lattice constants of 10.102 bohrs for silicon and 7.275 bohrs (with aspect ratio $c / a=1.046$ ) for $\mathrm{PbTiO}_{3}$. We use the dynamical quadrupole tensors computed in Refs. [21,32]. The phonon dispersions and e-ph perturbation potentials on coarse $\boldsymbol{q}$ point grids are computed with DFPT [15]. We employ the 
PERTURBO code [9] to compute the e-ph matrix elements on coarse Brillouin zone grids with $10 \times 10 \times 10 \boldsymbol{k}$ and $\boldsymbol{q}$ points for silicon and $8 \times 8 \times 8 \boldsymbol{k}$ and $\boldsymbol{q}$ points for $\mathrm{PbTiO}_{3}$. The Wannier functions are computed with the WANNIER 90 code [28] and employed in PERTURBO [9] to interpolate the short-range e-ph matrix elements.

We compute the scattering rates and electron mobility using the PERTURBO code [9]. Briefly, the band- and $\boldsymbol{k}$-dependent e-ph scattering rate $\Gamma_{n k}$ is obtained as

$$
\begin{aligned}
\Gamma_{n \boldsymbol{k}}= & \frac{2 \pi}{\hbar} \sum_{m v \boldsymbol{q}}\left|g_{m n v}(\boldsymbol{k}, \boldsymbol{q})\right|^{2} \\
& \times\left[\left(N_{v \boldsymbol{q}}+1-f_{m \boldsymbol{k}+\boldsymbol{q}}\right) \delta\left(\varepsilon_{n \boldsymbol{k}}-\varepsilon_{m \boldsymbol{k}+\boldsymbol{q}}-\hbar \omega_{\nu \boldsymbol{q}}\right)\right. \\
& \left.+\left(N_{v \boldsymbol{q}}+f_{m \boldsymbol{k}+\boldsymbol{q}}\right) \delta\left(\varepsilon_{n \boldsymbol{k}}-\varepsilon_{m \boldsymbol{k}+\boldsymbol{q}}+\hbar \omega_{\nu \boldsymbol{q}}\right)\right],
\end{aligned}
$$

where $\varepsilon_{n k}$ and $\hbar \omega_{\nu q}$ are the electron and phonon energies, respectively, and $f_{n k}$ and $N_{v q}$ are the corresponding temperaturedependent occupations. The scattering rate can be further divided into the long-range part [4] $\Gamma_{n k}^{\mathrm{L}}$ by replacing $|g|^{2}$ in Eq. (11) with $\left|g^{\mathrm{L}}\right|^{2}$. The carrier mobility is computed using $\mu=\sigma /\left(n_{c} e\right)$, where $\sigma$ is the electrical conductivity and $n_{c}$ is the carrier concentration. The electrical conductivity $\sigma$ is computed within the relaxation time approximation of the Boltzmann transport equation [9,33]:

$$
\sigma_{\alpha \beta}=e^{2} \int_{-\infty}^{+\infty} d E(-\partial f / \partial E) \Sigma_{\alpha \beta}(E, T),
$$

where $\Sigma_{\alpha \beta}(E, T)$ is the transport distribution function at energy $E$,

$$
\Sigma_{\alpha \beta}(E, T)=\frac{s}{\mathcal{N}_{k} \Omega} \sum_{n k} \tau_{n k}(T) v_{n k}^{\alpha} v_{n k}^{\beta} \delta\left(E-\varepsilon_{n k}\right),
$$

which is computed in PERTURBO using the tetrahedron integration method [34]. Above, $s$ is the spin degeneracy, $\mathcal{N}_{\boldsymbol{k}}$ is the number of $\boldsymbol{k}$ points, $v_{n k}$ is the band velocity, and $\tau_{n k}=\left(\Gamma_{n k}\right)^{-1}$ is the relaxation time. The mobility is computed with nondegenerate electron concentrations of $10^{15} \mathrm{~cm}^{-3}$ for silicon and $10^{17} \mathrm{~cm}^{-3}$ for $\mathrm{PbTiO}_{3}$. To fully converge the scattering rates and mobility, we use e-ph matrix elements evaluated on fine Brillouin zone grids with $200 \times 200 \times 200 k$ points and $8 \times 10^{6}$ random $\boldsymbol{q}$ points [35].

\section{RESULTS}

\section{A. Quadrupole effect on the e-ph matrix elements}

The long-range quadrupole e-ph interaction is present in a wide range of semiconductors and insulators, where the atomic dynamical quadrupoles are in general nonzero. We illustrate this point by studying silicon, a simple nonpolar semiconductor in which the Born charges-and thus the Fröhlich interaction - vanish and the presence of long-range interactions is not immediately obvious. Figure 2 shows the e-ph coupling strength $D_{\text {tot }}^{v}(\boldsymbol{q})$ in Eq. (10), computed directly using DFPT as a benchmark and compared with Wannier interpolation with and without inclusion of the quadrupole term. The DFPT benchmark e-ph matrix elements for optical modes approach a constant value as $\boldsymbol{q} \rightarrow 0$, as we show for the LO mode in the $\Gamma-L$ direction and the transverse optical (TO) mode along $\Gamma-K$. This trend is distinctive of

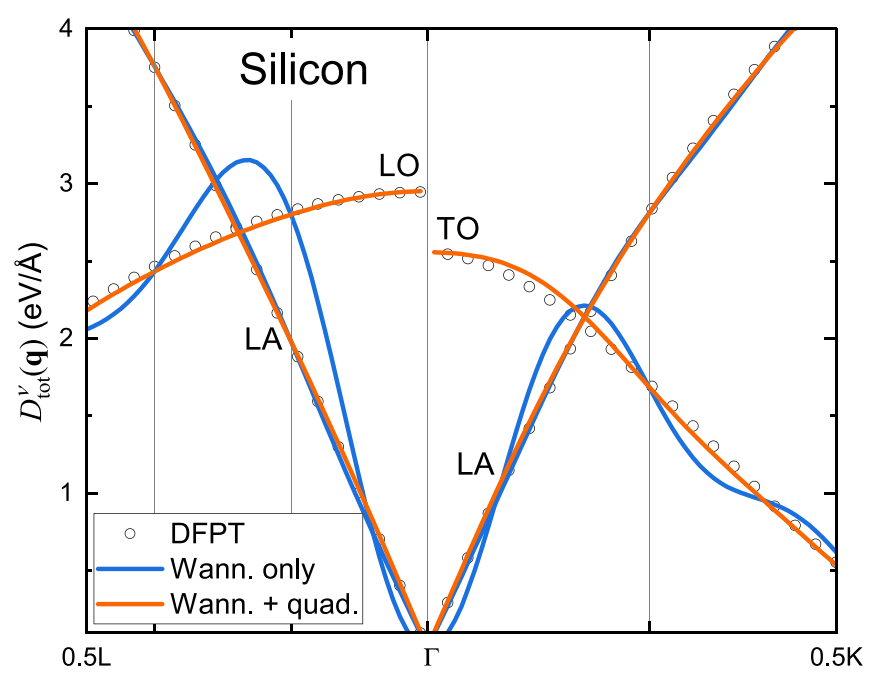

FIG. 2. Mode-resolved e-ph coupling strength [see Eq. (10)] in silicon, computed using the lowest valence band. The electron momentum $\boldsymbol{k}$ is fixed at the $\Gamma$ point and the phonon wave-vector $\boldsymbol{q}$ is varied along high-symmetry lines in the Brillouin zone. Benchmark results from DFPT (black circles) are compared with Wannier interpolation with the quadrupole e-ph interaction included (orange line) or neglected (blue line). The coarse-grid $\boldsymbol{q}$ points are indicated with vertical lines.

the quadrupole e-ph interaction, which is of order $q^{0}$ in the long-wavelength limit.

If the quadrupole term is neglected and all e-ph interactions are treated as short ranged, the e-ph matrix elements for optical modes in silicon incorrectly vanish as $\boldsymbol{q} \rightarrow 0$. The interpolated values for optical modes are underestimated between the $\Gamma$ point, where the error is greatest, and its nearest-neighbor $\boldsymbol{q}$ points in the coarse grid, where the error vanishes. Outside this $\boldsymbol{q}$-point region close to $\Gamma$, the interpolated matrix elements without the quadrupole interaction still deviate from the DFPT result, although the error is smaller than near $\Gamma$. When the quadrupole term is included, the long-range e-ph interactions for the optical modes are captured correctly, as can be seen for the Wannier plus quadrupole curves in Fig. 2. The rootmean-square deviation of $D_{\text {tot }}^{v}(\boldsymbol{q})$ from DFPT, for the optical branches shown in Fig. 2, is $0.78 \mathrm{eV} / \AA$ when the quadrupole term is neglected versus $0.03 \mathrm{eV} / \AA$ when the quadrupole term is included in the interpolation. This result highlights the importance of the quadrupole term to correctly describe longrange e-ph interactions in nonpolar semiconductors.

Observe also how for acoustic modes in silicon the quadrupole term has a nearly negligible effect, as we show for the longitudinal acoustic (LA) mode in Fig. 2. As contracting the dynamical quadrupoles $\mathbf{Q}_{\kappa}$ with a rigid shift of the lattice leads to a vanishing quadrupole contribution [18], one can obtain the quadrupole acoustic sum rule $\sum_{\alpha} Q_{\kappa, \alpha \beta \gamma}=0$ for nonpolar materials [18]. This sum rule, which is satisfied by the dynamical quadrupole values we employ for silicon [21], leads to a negligible quadrupole correction for acoustic modes in the long-wavelength limit. Though we focus on silicon in this work, on the basis of our results we expect sizable quadrupole contributions for optical modes, and negligible for acoustic modes, in all nonpolar semiconductors. 


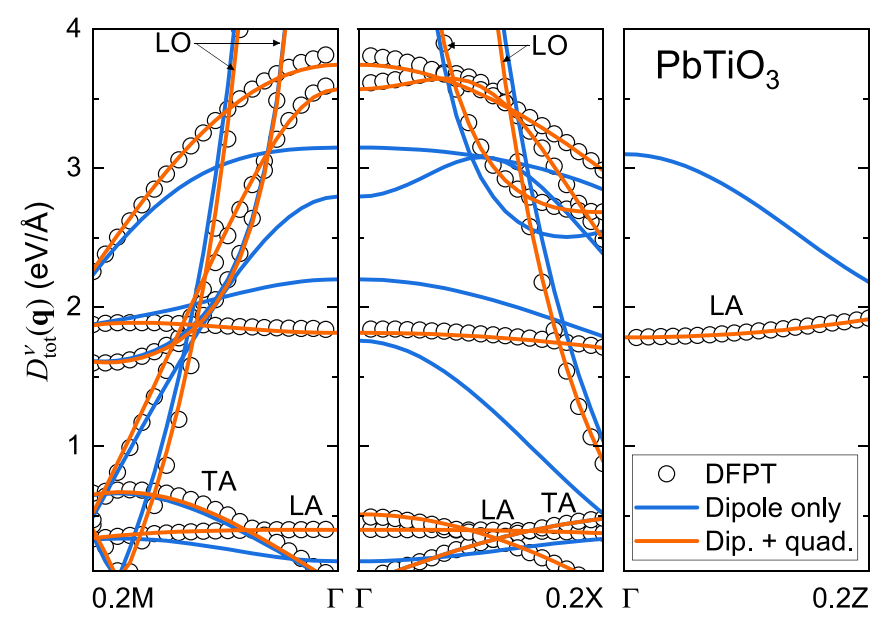

FIG. 3. Mode-resolved e-ph coupling strength [see Eq. (10)] in tetragonal $\mathrm{PbTiO}_{3}$, computed for the lowest conduction band. The initial electron momentum is fixed at the $\Gamma$ point and the phonon wave-vector $\boldsymbol{q}$ is varied along high-symmetry lines in the Brillouin zone. Benchmark results from DFPT (black circles) are compared with Wannier interpolation plus the Fröhlich interaction (blue line) and Wannier interpolation plus the Fröhlich and quadrupole interactions (orange line). Note that two LO branches with coupling strength exceeding the $y$-axis limit are not shown.

The quadrupole e-ph interaction is particularly critical in piezoelectric materials, as discussed here for tetragonal $\mathrm{PbTiO}_{3}$, a prototypical piezoelectric insulator. Piezoelectric materials are polar noncentrosymmetric systems with nonzero Born charges. As a result, while the dipole Fröhlich interaction is dominant for LO modes near $\boldsymbol{q} \rightarrow 0$ due to its $1 / q$ divergence, the quadrupole contribution is expected to be important for TO and acoustic modes (the quadrupole acoustic sum rule does not hold for polar noncentrosymmetric crystals).

Figure 3 shows the e-ph coupling strength $D_{\text {tot }}^{v}(\boldsymbol{q})$ in Eq. (10) for the DFPT benchmark in tetragonal $\mathrm{PbTiO}_{3}$, and compares it with interpolated results that include only the Fröhlich dipole interaction or both the Fröhlich and the quadrupole interactions. The short-range interactions are included through Wannier interpolation in both cases. When only the Fröhlich dipole interaction is included, the e-ph matrix elements deviate dramatically from the DFPT results. The values are either overestimated or underestimated depending on the phonon mode considered, with deviations from DFPT that depend strongly on the direction in which $\boldsymbol{q}$ approaches $\Gamma$ due to the nonanalytic character of the long-range e-ph interactions. When the quadrupole e-ph interaction is taken into account, the interpolated e-ph coupling strength matches the DFPT result very accurately for all phonon modes. For LO modes, the quadrupole correction is moderate due to the dominant Fröhlich term near $\boldsymbol{q}=0$. For other optical and acoustic modes with a finite e-ph coupling at $\boldsymbol{q}=0$, the quadrupole term removes the large error in the dipole-only results (up to an order of magnitude) and gives e-ph matrix elements in nearly exact agreement with DFPT. For the branches shown in Fig. 3, the root-mean-square deviation of $D_{\text {tot }}^{v}(\boldsymbol{q})$ from DFPT is $0.46 \mathrm{eV} / \AA$ for dipole-only results versus $0.03 \mathrm{eV} / \AA$ for our dipole plus quadrupole interpolation scheme. It is clear that
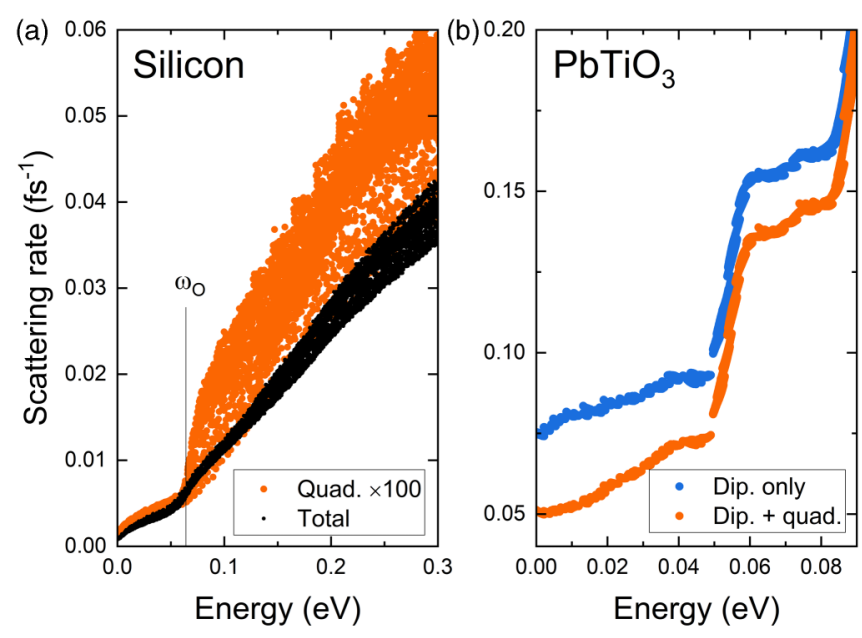

FIG. 4. Room temperature scattering rate versus electron energy (referenced to the conduction band minimum) in (a) silicon and (b) $\mathrm{PbTiO}_{3}$. For silicon we plot the quadrupole contribution multiplied by 100 (orange) and the total scattering rate (black), which includes the short-range and the quadrupole contributions. For $\mathrm{PbTiO}_{3}$ we show the long-range scattering rate computed using only the Fröhlich interaction (blue) or both the Fröhlich and quadrupole interactions (orange).

the quadrupole term is essential in piezoelectric materials for all phonon modes.

Contrary to silicon and nonpolar materials, the quadrupole term has a large effect for acoustic modes in piezoelectric materials, where it is one of the two contributions to the so-called piezoelectric e-ph interaction [24]. Expanding the phonon eigenvectors at $\boldsymbol{q} \rightarrow 0$ as $\mathbf{e}_{v \boldsymbol{q}} \approx \mathbf{e}_{v \boldsymbol{q}}^{(0)}+i \boldsymbol{q} \cdot \mathbf{e}_{v \boldsymbol{q}}^{(1)}$, one finds two contributions of order $q^{0}$ [18]. One is from the Born charges, and it reads $\mathbf{Z}_{\kappa} \mathbf{e}_{v q}^{(1)}$, a dipolelike interaction generated by atoms with a net charge experiencing different displacements due to strain from an acoustic mode. The other is the term $\mathbf{Q}_{\kappa} \mathbf{e}_{v q}^{(0)}$, from the dynamical quadrupoles, which is associated with a clamped-ion electronic polarization [36]. The $a b$ initio Fröhlich interaction includes only the former term, namely the strain component of the piezoelectric e-ph interaction, and thus the dipole-only scheme leads to large errors for acoustic phonons in $\mathrm{PbTiO}_{3}$ (see Fig. 3) as it neglects the important electronic quadrupole contribution. Until now, the $a b$ initio Fröhlich term has been mistakenly thought to fully capture piezoelectric e-ph interactions. Our results demonstrate that both dipole and quadrupole terms are essential for accurate acoustic mode e-ph interactions in piezoelectric materials [37]. The relative magnitude of the strain and quadrupole contributions is material dependentthe two terms can nearly cancel each other out, as we have shown elsewhere for GaN [29], or their ratio can be mode and phonon wave-vector dependent, as we find in $\mathrm{PbTiO}_{3}$.

\section{B. Quadrupole contribution to the scattering rate}

Because the quadrupole interaction has a significant effect on the e-ph matrix elements, we expect that it also plays a role in calculations of the e-ph scattering rate and mobility. Figure 4(a) shows both the quadrupole contribution and the 
total e-ph scattering rate in silicon at $300 \mathrm{~K}$ for electron energies near the conduction band minimum. We find that the quadrupole contribution to the scattering rate is about $1 \%$ of the total scattering rate at temperatures between $100-400 \mathrm{~K}$. At electron energies below the optical phonon emission threshold in silicon $\left(\hbar \omega_{\mathrm{O}} \approx 65 \mathrm{meV}\right.$ relative to the conduction band minimum), absorption and emission of acoustic phonons dominate the scattering processes, and thus we find a small correction due to the quadrupole interaction, which minimally affects acoustic modes in silicon. Since the quadrupole acoustic sum rule holds only in the long wavelength limit, the quadrupole interaction can still contribute to finite- $\boldsymbol{q}$ acoustic scattering, as is shown by the fact that the quadrupole scattering rate at energy below $\hbar \omega_{\mathrm{O}}$ is proportional to the total scattering rate. The quadrupole contribution increases sharply above the optical emission threshold because the quadrupole term is greater for optical modes in silicon. For the same reason, the relative contribution of the quadrupole term increases slightly with temperature in the $100-400 \mathrm{~K}$ range, varying from $1 \%$ of the total scattering rate at $100 \mathrm{~K}$ to $1.5 \%$ at $400 \mathrm{~K}$.

The effect of the quadrupole interaction on the scattering rates is greater in $\mathrm{PbTiO}_{3}$. Our analysis focuses on the eph scattering rate due to the long-range e-ph interactions, although similar conclusions hold for the total scattering rate. Figure 4(b) shows the long-range e-ph scattering rate in $\mathrm{PbTiO}_{3}$ at $300 \mathrm{~K}$ as a function of electron energy, comparing results that include only the dipole Fröhlich interaction with results from our approach including both the dipole and quadrupole terms. The scattering rate from the long-range e-ph interactions is lower at all energies when the quadrupole term is taken into account. The difference is greatest near the band edge, where the scattering rate due to the dipole interaction alone is $0.075 \mathrm{fs}^{-1}$ versus a $50 \%$ smaller value of $0.050 \mathrm{fs}^{-1}$ for dipole plus quadrupole.

These trends can be understood on the basis of the e-ph matrix element analysis in Fig. 3. The errors found when neglecting the quadrupole term in $D_{\mathrm{tot}}^{v}(\boldsymbol{q})$, which is proportional to the absolute value of the matrix elements [see Eq. (10)], are amplified in calculations of the scattering rate, which is proportional to the square of the matrix elements. The largest errors we find for $D_{\mathrm{tot}}^{v}(\boldsymbol{q})$ are in the $\boldsymbol{q} \rightarrow 0$ limit, especially for the acoustic modes. For example, for the LA mode in the $\Gamma-M$ and $\Gamma-X$ directions, the value of $D_{\text {tot }}^{v}(\boldsymbol{q})$ from the dipole-only calculation is $0.17 \mathrm{eV} / \AA ̊ \AA$ compared to a twice-greater value of $0.40 \mathrm{eV} / \AA$ when the quadrupole term is included. This leads to a fourfold increase of the LA mode scattering rate upon including the quadrupole interaction. Opposite to the silicon case, in $\mathrm{PbTiO}_{3}$ the relative magnitude of the quadrupole correction is greater at lower temperatures because the quadrupole interaction is stronger for acoustic modes. Near the band edge, we find quadrupole corrections to the longrange scattering rate ranging from $97 \%$ at $100 \mathrm{~K}$ to $38 \%$ at $400 \mathrm{~K}$. Given that low-energy electronic states near the band edge govern transport properties, including the quadrupole term is critical to accurately computing electronic transport.

\section{Quadrupole contribution to the mobility}

The effect of the quadrupole e-ph interaction on the mobility is noteworthy. Figure 5(a) shows the temperature-
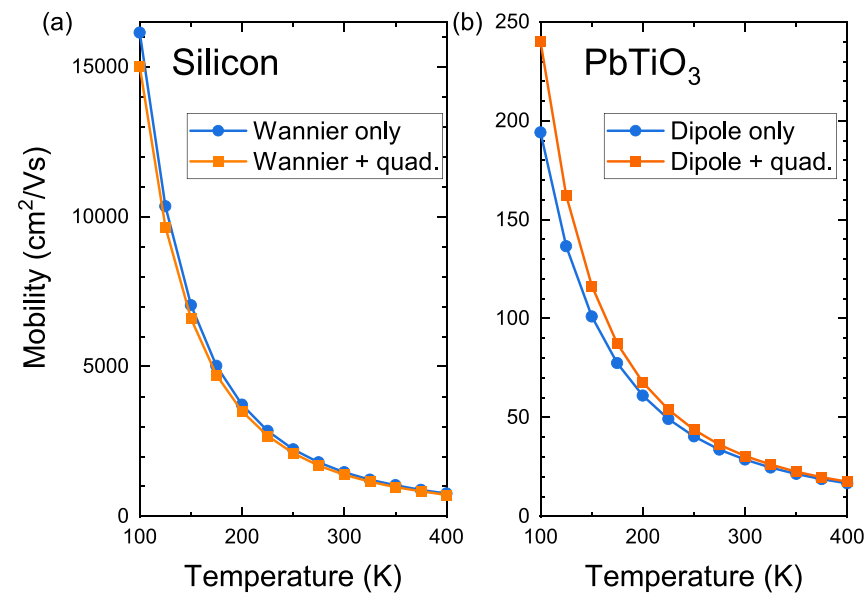

FIG. 5. Computed temperature-dependent electron mobility in (a) silicon and (b) tetragonal $\mathrm{PbTiO}_{3}$. The plot compares the mobility obtained when the quadrupole e-ph interaction is included (orange squares) or neglected (blue circles). The $\mathrm{PbTiO}_{3}$ results are for transport in the basal $x y$ plane.

dependent electron mobility in silicon computed with and without the quadrupole term. Including the quadrupole interaction reduces the computed mobility by approximately 5\%-10\% due to the increased e-ph coupling strength and scattering rates. For example, the computed mobility at $300 \mathrm{~K}$ is $1390 \mathrm{~cm}^{2} / \mathrm{V}$ s when including the quadrupole interaction versus a value of $1473 \mathrm{~cm}^{2} / \mathrm{V} \mathrm{s}$ with the conventional interpolation approach in which all e-ph interactions in silicon are treated as short ranged. This discrepancy is due to the underestimation of the e-ph coupling strength for optical modes in the conventional approach, especially at small values of $\boldsymbol{q}$ as shown in Fig. 2.

In silicon, e-ph scattering mediates both intravalley and intervalley processes. The quadrupole interaction affects mainly small- $\boldsymbol{q}$ intravalley processes associated with optical phonons. However, intravalley processes-particularly those associated with acoustic phonons-are dominant only at low temperature, while at higher temperatures intervalley processes mediated by large- $\boldsymbol{q}$ phonons are dominant [38]. As a result, the intravalley optical phonon scattering processes mediated by the quadrupole interaction are active mainly at low temperature and are overall weaker than other scattering contributions in silicon, including acoustic intravalley scattering at low temperature. The contribution of the quadrupole correction to the mobility is thus maximal at low temperature and overall relatively small.

Although we focus on silicon, we expect that these trends apply in general to nonpolar semiconductors because small$\boldsymbol{q}$ optical e-ph coupling will consistently be underestimated without the quadrupole term. The long-range quadrupole eph interaction is thus surprisingly manifest in the transport properties of nonpolar materials.

We find an opposite trend in $\mathrm{PbTiO}_{3}$, in which including the quadrupole interaction increases the mobility by $10 \%-$ $25 \%$ between 100-400 K, as seen in Fig. 5(b). The quadrupole term gives a larger correction at lower temperatures, reaching values up to $\sim 25 \%$ at $100 \mathrm{~K}$. This result is due to the dominant acoustic mode contribution at low temperatures together with 
the large quadrupole correction for acoustic modes in piezoelectric materials. At higher temperatures, where optical mode scattering is dominant and acoustic scattering less important, the quadrupole contribution is smaller, only about $10 \%$ at $400 \mathrm{~K}$. Due to differences in the quadrupole interaction for different phonon modes and to varying mode contributions to the mobility as a function of temperature, including the quadrupole term corrects the temperature dependence of the mobility [29] and is essential in piezoelectric materials.

\section{DISCUSSION}

We briefly discuss a technical aspect of the e-ph matrix element interpolation. The treatment of long-wavelength perturbations with wave-vector $\boldsymbol{q} \rightarrow 0$ in DFPT is critical in semiconductors and insulators [16,39]. The lattice-periodic part of the phonon perturbation potential $\Delta v_{q}(\mathbf{r})$ is the sum of a Coulomb and an exchange-correlation contribution,

$$
\Delta v_{\boldsymbol{q}}(\mathbf{r})=\Delta v_{\boldsymbol{q}, \mathrm{C}}(\mathbf{r})+\Delta v_{\boldsymbol{q}, \mathrm{XC}}(\mathbf{r}) .
$$

The Coulomb contribution $\Delta v_{q, \mathrm{C}}(\mathbf{r})$ combines the variation of the Hartree and electron-nuclei interactions. Its integral over the unit cell [39]

$$
\Delta(\boldsymbol{q})=\frac{1}{\Omega} \int_{\Omega} d \mathbf{r} v_{\boldsymbol{q}, \mathrm{C}}(\mathbf{r})
$$

is well behaved for insulators (and semiconductors) at finite $\boldsymbol{q}$ values, but is ill defined at $\boldsymbol{q}=0$. First-principles codes such as QUANTUM ESPRESSO [30] subtract $\Delta(\boldsymbol{q})$ from the perturbation potential at $\boldsymbol{q}=0$, thus making it discontinuous at $\boldsymbol{q}=0$. Therefore, due to both the discontinuity at $\boldsymbol{q}=0$ and the nonanalytic behavior near $\boldsymbol{q}=0$, the e-ph matrix elements are challenging to interpolate in the long-wavelength limit.

In our scheme we identify the quadrupole interaction as the key long-range term in nonpolar materials, and remove the nonanalytic behavior near $\boldsymbol{q}=0$ on the coarse grid by subtracting the quadrupole term. This strategy improves the interpolation near $\boldsymbol{q}=0$ in nonpolar materials, at once capturing the correct physics and smoothing the coarse-grid matrix element to be interpolated. Due to the nonanalytic behavior, denser DFPT grids cannot fully remove the interpolation error if the quadrupole term is not subtracted on the coarse grid [40]. For polar materials such as $\mathrm{PbTiO}_{3}$, the nonanalytic behavior is due to both the dipole (Fröhlich) and quadrupole long-range e-ph interactions. By subtracting both terms in our scheme in polar materials, the coarse-grid matrix elements to be interpolated are made smooth and the interpolation approach more reliable. The nonanalytic behavior of the Coulomb potential is correctly reconstructed by adding back the dipole (in polar materials) and quadrupole (in all insulators) contributions after interpolation.

\section{CONCLUSION}

In summary, we developed an accurate approach for computing the quadrupole e-ph interaction from first principles. This advance resolves the outstanding problem of correctly quantifying long-range e-ph interactions for all phonon modes in semiconductors and insulators. Our results clearly show that the quadrupole interactions are crucial for obtaining accurate e-ph matrix elements, scattering rates, and electronic transport properties. The quadrupole effect is particularly apparent in piezoelectric materials such as wurtzite GaN [29] and $\mathrm{PbTiO}_{3}$, in which neglecting the quadrupole interaction leads to large and uncontrolled errors. The method introduced in this work enables accurate calculations of electrical transport, thermoelectric properties and superconductivity in a wide range of materials.

Note added. Recently, we became aware of a related work by another group that reaches similar conclusions about the importance of the dynamical quadrupole term to obtain an accurate physical description of e-ph interactions $[40,41]$.

\section{ACKNOWLEDGMENTS}

J.P. acknowledges support by the Korea Foundation for Advanced Studies. V.A.J. thanks the Resnick Sustainability Institute at Caltech for fellowship support. This work was supported by the National Science Foundation under Grants No. DMR-1750613 for theory development and No. ACI1642443 for code development. J.-J.Z. acknowledges partial support from the Joint Center for Artificial Photosynthesis, a DOE Energy Innovation Hub, as follows: the development of some computational methods employed in this work was supported through the Office of Science of the US Department of Energy under Award No. DE-SC0004993. C.E.D. acknowledges support from the National Science Foundation under Grant No. DMR-1918455. The Flatiron Institute is a division of the Simons Foundation. This research used resources of the National Energy Research Scientific Computing Center, a DOE Office of Science User Facility supported by the Office of Science of the US Department of Energy under Contract No. DE-AC02-05CH11231.
[1] J. M. Ziman, Electrons and Phonons: The Theory of Transport Phenomena in Solids (Oxford University Press, Oxford, 2001).

[2] M. Bernardi, Eur. Phys. J. B 89, 239 (2016).

[3] M. Bernardi, D. Vigil-Fowler, J. Lischner, J. B. Neaton, and S. G. Louie, Phys. Rev. Lett. 112, 257402 (2014).

[4] J.-J. Zhou and M. Bernardi, Phys. Rev. B 94, 201201(R) (2016).

[5] V. A. Jhalani, J.-J. Zhou, and M. Bernardi, Nano Lett. 17, 5012 (2017).

[6] N.-E. Lee, J.-J. Zhou, L. A. Agapito, and M. Bernardi, Phys. Rev. B 97, 115203 (2018).
[7] J.-J. Zhou, O. Hellman, and M. Bernardi, Phys. Rev. Lett. 121, 226603 (2018).

[8] J.-J. Zhou and M. Bernardi, Phys. Rev. Res. 1, 033138 (2019).

[9] J.-J. Zhou, J. Park, I.-T. Lu, I. Maliyov, X. Tong, and M. Bernardi, arXiv:2002.02045.

[10] W. Li, Phys. Rev. B 92, 075405 (2015).

[11] T.-H. Liu, J. Zhou, B. Liao, D. J. Singh, and G. Chen, Phys. Rev. B 95, 075206 (2017).

[12] J. Ma, A. S. Nissimagoudar, and W. Li, Phys. Rev. B 97, 045201 (2018). 
[13] T. Sohier, D. Campi, N. Marzari, and M. Gibertini, Phys. Rev. Mater. 2, 114010 (2018).

[14] R. M. Martin, Electronic Structure: Basic Theory and Practical Methods (Cambridge University Press, Cambridge, UK, 2004).

[15] S. Baroni, S. de Gironcoli, A. Dal Corso, and P. Giannozzi, Rev. Mod. Phys. 73, 515 (2001).

[16] L. A. Agapito and M. Bernardi, Phys. Rev. B 97, 235146 (2018).

[17] P. Lawaetz, Phys. Rev. 183, 730 (1969).

[18] P. Vogl, Phys. Rev. B 13, 694 (1976).

[19] M. Stengel, Phys. Rev. B 88, 174106 (2013).

[20] C. E. Dreyer, M. Stengel, and D. Vanderbilt, Phys. Rev. B 98, 075153 (2018).

[21] M. Royo and M. Stengel, Phys. Rev. X 9, 021050 (2019).

[22] J. Sjakste, N. Vast, M. Calandra, and F. Mauri, Phys. Rev. B 92, 054307 (2015).

[23] C. Verdi and F. Giustino, Phys. Rev. Lett. 115, 176401 (2015).

[24] G. D. Mahan, Condensed Matter in a Nutshell (Princeton University Press, Princeton, NJ, 2011).

[25] H. Fröhlich, Adv. Phys. 3, 325 (1954).

[26] M. Born and K. Huang, Dynamical Theory of Crystal Lattices (Oxford University Press, Oxford, 1954).

[27] D. J. Griffiths, Introduction to Electrodynamics (Cambridge University Press, Cambridge, 2017).

[28] G. Pizzi, V. Vitale, R. Arita, S. Blügel, F. Freimuth, G. Géranton, M. Gibertini, D. Gresch, C. Johnson, T. Koretsune, J. Ibañez-Azpiroz, H. Lee, J.-M. Lihm, D. Marchand, A. Marrazzo, Y. Mokrousov, J. I. Mustafa, Y. Nohara, Y. Nomura, L. Paulatto, S. Poncé, T. Ponweiser, J. Qiao, F. Thöle, S. S. Tsirkin, M. Wierzbowska, N. Marzari, D. Vanderbilt, I. Souza, A. A. Mostofi, and J. R. Yates, J. Phys.: Condens. Matter 32, 165902 (2020).

[29] V. A. Jhalani, J.-J. Zhou, J. Park, C. E. Dreyer, and M. Bernardi, Phys. Rev. Lett. 125, 136602 (2020).

[30] P. Giannozzi, S. Baroni, N. Bonini, M. Calandra, R. Car, C. Cavazzoni, D. Ceresoli, G. L. Chiarotti, M. Cococcioni, I. Dabo, A. D. Corso, S. de Gironcoli, S. Fabris, G. Fratesi, R. Gebauer, U. Gerstmann, C. Gougoussis, A. Kokalj, M. Lazzeri, L. Martin-Samos, N. Marzari, F. Mauri, R. Mazzarello, S. Paolini, A. Pasquarello, L. Paulatto, C. Sbraccia, S. Scandolo, G. Sclauzero, A. P. Seitsonen, A. Smogunov, P. Umari, and R. M. Wentzcovitch, J. Phys.: Condens. Matter 21, 395502 (2009).
[31] M. J. van Setten, M. Giantomassi, E. Bousquet, M. J. Verstraete, D. R. Hamann, X. Gonze, and G. M. Rignanese, Comput. Phys. Commun. 226, 39 (2018).

[32] X. Gonze, B. Amadon, G. Antonius, F. Arnardi, L. Baguet, J.-M. Beuken, J. Bieder, F. Bottin, J. Bouchet, E. Bousquet, N. Brouwer, F. Bruneval, G. Brunin, T. Cavignac, J.-B. Charraud, W. Chen, M. Côté, S. Cottenier, J. Denier, G. Geneste, P. Ghosez, M. Giantomassi, Y. Gillet, O. Gingras, D. R. Hamann, G. Hautier, X. He, N. Helbig, N. Holzwarth, Y. Jia, F. Jollet, W. Lafargue-Dit-Hauret, K. Lejaeghere, M. A. L. Marques, A. Martin, C. Martins, H. P. C. Miranda, F. Naccarato, K. Persson, G. Petretto, V. Planes, Y. Pouillon, S. Prokhorenko, F. Ricci, G.-M. Rignanese, A. H. Romero, M. M. Schmitt, M. Torrent, M. J. van Setten, B. Van Troeye, M. J. Verstraete, G. Zérah, and J. W. Zwanziger, Comput. Phys. Commun. 248, 107042 (2020).

[33] G. D. Mahan, Many-Particle Physics, 3rd ed. (Springer, New York, 2000).

[34] P. E. Blöchl, O. Jepsen, and O. K. Andersen, Phys. Rev. B 49, 16223 (1994).

[35] Uniform grids and random sampling can both be used to converge the scattering rate as long as a sufficiently large number of $\boldsymbol{q}$ points is employed. In our experience, random sampling, which corresponds to Monte Carlo integration, usually leads to slightly faster convergence than uniform grids. It also has the advantage that one can systematically improve the convergence by running additional calculations, thus improving the sampling. For comparison, checking convergence with uniform grids requires using increasingly denser uniform meshes, which is computationally more expensive and inconvenient.

[36] F. Bernardini, V. Fiorentini, and D. Vanderbilt, Phys. Rev. B 56, R10024 (1997).

[37] G. Sághi-Szabó, R. E. Cohen, and H. Krakauer, Phys. Rev. Lett. 80, 4321 (1998).

[38] J. Park, J.-J. Zhou, and M. Bernardi, Phys. Rev. B 101, 045202 (2020).

[39] M. Calandra, G. Profeta, and F. Mauri, Phys. Rev. B 82, 165111 (2010).

[40] G. Brunin, H. P. C. Miranda, M. Giantomassi, M. Royo, M. Stengel, M. J. Verstraete, X. Gonze, G.-M. Rignanese, and G. Hautier, Phys. Rev. Lett. 125, 136601 (2020).

[41] G. Brunin, H. P. C. Miranda, M. Giantomassi, M. Royo, M. Stengel, M. J. Verstraete, X. Gonze, G.-M. Rignanese, and G. Hautier, Phys. Rev. B 102, 094308 (2020). 